\title{
CERTAIN CLASSES OF MEROMORPHIC MULTIVALENT FUNCTIONS
}

\author{
B. A. URALEGADDI AND C. SOMANATHA
}

\begin{abstract}
Let $f(z)=\frac{1}{z^{p}}+\sum_{n=1}^{\infty} \frac{a_{n-1}}{z^{p-n}}$ be regular in the punctured disk $E=\{z: 0<|z|<1\}$ and $D^{n+p-1} f(z)=\frac{1}{z^{p}(1-z)^{n+p}} * f(z)$ where * denotes the Hadamard product and $n$ is any integer greater than $-p$. For $-1 \leq B<A \leq 1$, let $C_{n, p}(A, B)$ denote the class of functions $f(z)$ satisfying

$$
-z^{p+1}\left(D^{n+p-1} f(z)\right)^{\prime} \prec p \frac{1+A z}{1+B z}, \quad|z|<1 .
$$

This paper establishes the property $C_{n+1, p}(A, B) \subset C_{n, p}(A, B)$. Further property preserving integral operators, coefficient inequalities and a closure theorem for these classes are obtained. Our results generalise some of the recent results of Ganigi and Uralegaddi [1].
\end{abstract}

\section{Introduction}

Let $\sum_{p}$ denote the class of functions $f(z)=\frac{1}{z^{p}}+\frac{a_{0}}{z^{p-1}}+\frac{a_{1}}{z^{p-2}}+\cdots$, that are regular in the punctured disk $E=\{z: 0<|z|<1\}$ and $p$ a positive integer. Let $D^{n+p-1} f(z)=\frac{1}{z^{p}(1-z)^{n+p}} * f(z)$, where $n$ is any integer greater than $-p$ and the operation $*$ denotes the Hadamard product. A function $f$ of $\sum_{p}$ is said to be in the class $C_{n, p}(A, B)$ if

$$
-z^{p+1}\left(D^{n+p-1} f(z)\right)^{\prime} \prec p \frac{1+A z}{1+B z}, \quad z \varepsilon \triangle=\{z:|z|<1\} \text { where }
$$

Received July 20, 1991.

Keywords and phrases. Multivalent functions, Hadamard product, coefficient inequalities. AMS 1980 subject classifications primary $30 \mathrm{C} 45$ 
$-1 \leq B<A \leq 1$, and the symbol $\prec$ denotes subordination.

Equivalently, a function $f$ of $\sum_{p}$ belongs to $C_{n, p}(A, B)$ if and only if there exists a function $w$ regular in $\triangle$, satisfying $w(0)=0$ and $|w(z)|<1$ for $z \varepsilon \triangle$ such that

$$
-z^{p+1}\left(D^{n+p-1} f(z)\right)^{\prime}=p \frac{1+A w(z)}{1+B w(z)}
$$

It is easy to verify the identlty

$$
z\left(D^{n+p-1} f(z)\right)^{\prime}=(n+p) D^{n+p} f(z)-(n+2 p) D^{n+p-1} f(z) .
$$

Using (2), (1) may be written as

$$
-z^{p}\left[(n+p) D^{n+p} f(z)-(n+2 p) D^{n+p-1} f(z)\right]=p \frac{1+A w(z)}{1+B w(z)}
$$

This paper establishes the property $C_{n+1, p}(A, B) \subset C_{n, p}(A, B)$. Further we obtain class preserving integral operators, coefficient inequalities and a closure theorem for functions in these classes. By assigning speciffic values to $A$ and $B$ and putting $p=1$, we obtain some of the results in [1, Th.1 and Th.2].

2. The classes $C_{n, p}(A, B)$.

We shall prove the following.

Lemma. A function $f$ in $\sum_{p}$ belongs to the class $C_{n, p}(A, B),-1 \leq B<$ $A \leq 1$ if and only if

$$
\left|z^{p+1}\left(D^{n+p-1} f(z)\right)^{\prime}+m\right|<M, \quad z \varepsilon \triangle
$$

where

$$
m=p(1-A B) /\left(1-B^{2}\right) \text { and } M=p(A-B) /\left(1-B^{2}\right) .
$$

Proof is similar to that of lemma 2.1 in [3].

Theorem 1.

$$
C_{n+1, p}(A, B) \subset C_{n, p}(A, B), \quad n>-p .
$$


Proof. Let $f \varepsilon C_{n+1, p}(A, B)$. Suppose thăt

$$
-z^{p}\left[(n+p) D^{n+p} f(z)-(n+2 p) D^{n+p-1} f(z)\right]=p \frac{1+A w(z)}{1+B w(z)} .
$$

That is

$$
z^{p}\left[(n+p) D^{n+p} f(z)-(n+2 p) D^{n+p-1} f(z)\right]=-p \frac{1+A w(z)}{1+B w(z)} .
$$

where $w$ is either regular or meromorphic in $\triangle$. clearly $w(0)=0$ becasuce the left side of (6) is $-p$ when $z=0$. Differentiating (6) and using (1) we obtain

$$
z^{p+1}\left(D^{n+p} f(z)\right)^{\prime}=-p \frac{1+A w(z)}{1+B w(z)}-p\left(\frac{A-B}{n+p}\right)\left[\frac{z w^{\prime}(z)}{(1+B w(z))^{2}}\right] .
$$

Hence

$$
\begin{aligned}
z^{p+1}\left(D^{n+p} f(z)\right)^{\prime}+m= & \frac{(m-p)-(A p-B m) w(z)}{1+B w(z)} \\
& -p\left(\frac{A-B}{n+p}\right)\left[\frac{z w^{\prime}(z)}{(1+B w(z))^{2}}\right] .
\end{aligned}
$$

Let $r^{*}$ be the distance from the origin to the nearest pole of $w$ in $\triangle$. Then $w$ is reqular in $|z|<r_{0}=\min \left\{r^{*}, 1\right\}$. By a lemma due to Jack [2] for $|z|<r\left(r<r_{0}\right)$ there exists a point $z_{0}$ such that

$$
z_{0} w^{\prime}\left(z_{0}\right)=k w\left(z_{0}\right), \quad k \geq 1
$$

From (7) and (8) we have

$$
z_{0}^{p+1}\left(D^{n+p} f\left(z_{0}\right)\right)^{\prime}+m=\frac{N\left(z_{0}\right)}{R\left(z_{0}\right)}
$$

where

$$
\begin{aligned}
N\left(z_{0}\right)= & (n+p)(m-p)+[(n+p)(B m-A p)+B(n+p)(m-p) \\
& -k p(A-B)] w\left(z_{0}\right)+B(n+p)(B m-A p) W^{2}\left(z_{0}\right)
\end{aligned}
$$

and

$$
R\left(z_{0}\right)=(n+p)\left(1+2 B w\left(z_{0}\right)+B^{2} W^{2}\left(z_{0}\right)\right]
$$


Now suppose if possible $\max _{|z|=r}|w(z)|=1$ for some $r, r<r_{0} \leq 1$. At the point $z_{0}$ where this occured, we would have $\left|w\left(z_{0}\right)\right|=1$. Then by using the identities

$$
p-m=B M \text { and } A p-B m=M
$$

we have

$$
\left|N\left(z_{0}\right)\right|^{2}-M^{2}\left|R\left(z_{0}\right)\right|^{2}=x+2 y \quad \operatorname{Re}\left\{W\left(z_{0}\right)\right\}
$$

where

$$
x=k p(A-B)\left\{k p(A-B)+2 M(n+p)\left(1+B^{2}\right)\right\}
$$

and

$$
y=2 k p(A-B) M B(n+p) .
$$

From (10) we have

$$
\left|N\left(z_{0}\right)\right|^{2}-M^{2}\left|R\left(z_{0}\right)\right|^{2}>0, \text { provided } x \pm 2 y>0
$$

Now

$$
x+2 y=k p(A-B)\left\{k p(A-B)+2 M(n+p)(1+B)^{2}\right\}>0
$$

and

$$
x-2 y=k p(A-B)\left\{k p(A-B)+2 M(n+p)(1-B)^{2}\right\}>0 .
$$

Thus it follows from (9) and (11) that

$$
\left|z_{0}^{p+1}\left(D^{n+p} f\left(z_{0}\right)\right)^{\prime}+m\right|>M .
$$

But, in view of the above lemma, this is a contradiction to the fact that $f \varepsilon C_{n+1, p}(A, B)$. So we cannot have $\left|w\left(z_{0}\right)\right|=1$. Thus $|w(z)| \neq 1$ in $|z|<r_{0}$. Since $w(0)=0,|r \cdot(z)|$ is continuous and $|w(z)| \neq 1$ in $|z|<r_{0}$, we cannot have a pole at $|z|=r$. Hence $w$ is regular in $\triangle$ and satisfies $|w(z)|<1$ for $z \varepsilon \triangle$. Therefore, from (o) it follows that $f \varepsilon C n, p(A, B)$.

In the next theorem we obtain property preserving integral operators for $C_{n, p}(A, B)$. 
Theorem 2. Let $p$ be a positive integer and $n$ is ariy integer greater than $-p$. If $f(z) \in C_{n, p}(A, B)$ ard $k \in(c \cdots p+1)>0$ then

$$
F(z)=\frac{c-p+1}{z^{c+1}} \int_{0}^{z} t^{C} f(t) d t \varepsilon C_{n, p}(A, B) .
$$

Proof. From the definition of $F$ defined by (12) we have

$$
z\left(D^{n+p-1} F(z)\right)^{\prime}=(c-p+1) D^{n+p-1} f(z)-(c+1) D^{n+p-1} F(z)
$$

Let us suppose that

$$
-z^{p+1}\left(D^{n+p-1} F(z)\right)^{\prime}=p \frac{1+A w(z)}{1+B w(z)}
$$

where the function $w$ is either regular or meromorphic in $\triangle$. Clearly $w(0)=0$. Elimiating $\left(D^{n+p-1} F(z)\right)^{\prime}$ from (13) and (14), we have

$$
(c-p+1) D^{n+p-1} f(z)=(c+1) D^{n+p-1} F(z)-p z^{-p}\left[\frac{1+A w(z)}{1+B w(z)}\right]
$$

Differentiating (15) and using (14), we obtain

$$
z^{p+1}\left(D^{n+p-1} f(z)\right)^{\prime}=-p \frac{1+A w(z)}{1+B w(z)}-p\left(\frac{A-B}{c-p+1}\right)\left[\frac{z w^{\prime}(z)}{(1+B w(z))^{2}}\right] .
$$

Hence

$$
\begin{aligned}
z^{p+1}\left(D^{n+p-1} f(z)\right)^{\prime}+m= & \frac{(m-p)-(A p-B m) w(z)}{1+B w(z)} \\
& -p\left(\frac{A-B}{c-p+1}\right)\left[\frac{z w^{\prime}(z)}{(1+B w(z))^{2}}\right]
\end{aligned}
$$

The remaining part of the proof is similar to that of theorem 1.

Remark. For $p=1, A=1-2 \alpha$ and $B=-1$ theorems 1 and 2 yield the earlier results in [1, Th.1 and Th.2]. 
Theorem 3. Let $p$ be a positive integer and $n$ is any integer such that $n>-p$ and $F(z)=(n+p) z^{-n-2 p} \int_{0}^{z} t^{n+2 p-1} f(t) d t$. Then $F \varepsilon C_{n+1, p}(A, B)$ if and only if $f \varepsilon C_{n, p}(A, B)$.

Proof. From the definition of $F$ we have

$$
\left(D^{n+p} F(z)\right)^{\prime}=\left(D^{n+p-1} f(z)\right)^{\prime}
$$

and the result follows.

Now we obtain coefficient inequalities for the class $C_{n, p}(A, B)$.

Theorem 4. Let $f(z)=\frac{1}{z^{p}}+\frac{a_{0}}{z^{p-1}}+\frac{a_{1}}{z^{p-2}}+\cdots$. If $f \varepsilon C_{n, p}(A, B)$, then

$$
\left|a_{k-1}\right| \leq \frac{p(A-B)}{(k-p) \alpha(n, k)}, \quad k=1,2, \ldots,
$$

where $\alpha(n, j)=\left(\begin{array}{c}n+p+j-1 \\ n+p-1\end{array}\right)$. Then result is sharp.

Proof. Since $f \varepsilon C_{n, p}(A, B)$, we have

$$
\left(D^{n+p-1} f(z)\right)^{\prime}=-p z^{-p-1} \frac{1+A w(z)}{1+B w(z)}
$$

where $w(z)=\sum_{j=1}^{\infty} t_{j} z^{j}$ is regular in $\triangle$ and $|w(z)|<1$ for $z \varepsilon \triangle$. Then

$$
\left(D^{n+p-1} f(z)\right)^{\prime}+p z^{-p-1}=-\left[A p z^{-p-1}+B\left(D^{n+p-1} f(z)\right)^{\prime}\right] w(z)
$$

or

$$
\begin{aligned}
& \sum_{j=1}^{\infty}(j-p) \alpha(n, j) a_{j-1} z^{-p+j-1} \\
= & -\left[(A-B) p z^{-p-1}+B \sum_{j=1}^{\infty}(j-p) \alpha(n, j) a_{j-1} z^{-p+j-1}\right] \sum_{j=1}^{\infty} t_{j} z^{j} .
\end{aligned}
$$

Comparing coefficients of like powers of $z$ on both the sides of (18) we see that the coefficient $a_{k-1}$ on the left side of (18) depends only on $a_{0}, a_{1}, \ldots, a_{k-3}, a_{k-2}$ 
on the right side of (18). Hence for $j=1,2, \ldots$, it follows from (18) that

$$
\begin{aligned}
& \sum_{j=1}^{k}(j-p) \alpha(n, j) a_{j-1} z^{-p+j-1}+\sum_{j=k}^{\infty} c_{j} z^{-p+j} \\
= & -\left[(A-B) p z^{-p-1}+B \sum_{j=1}^{k-1}(j-p) \alpha(n, j) a_{j-1} z^{-p+j-1}\right] w(z)
\end{aligned}
$$

where $c_{j}$ are some coumplex numbers. Since $|w(z)|<1$, by using parseval's identity we obtain

$$
\begin{aligned}
& \sum_{j=1}^{k}(j-p)^{2}(\alpha(n, j))^{2}\left|a_{j-1}\right|^{2} r^{2(-p+j-1)}+\sum_{j=k}^{\infty}\left|c_{j}\right|^{2} r^{2(-p+j)} \\
\leq & (A-B)^{2} p^{2} r^{2(-p-1)}+B^{2} \sum_{j=1}^{k-1}(j-p)^{2}(\alpha(n, j))^{2}\left|a_{j-1}\right|^{2} r^{2(-p+j-1)} \\
\leq & (A-B)^{2} p^{2}+B^{2} \sum_{j=1}^{k-1}(j-p)^{2}(\alpha(n, j))^{2}\left|a_{j-1}\right|^{2}
\end{aligned}
$$

Letting $r \rightarrow 1$ on the left side of this inequality we get

$$
\sum_{j=1}^{k}(j-p)^{2}(\alpha(n, j))^{2}\left|a_{j-1}\right|^{2} \leq(A-B)^{2} p^{2}+B^{2} \sum_{j=1}^{k-1}(j-p)^{2}(\alpha(n, j))^{2}\left|a_{j-1}\right|^{2}
$$

Thus

$$
\begin{aligned}
(k-p)^{2}(\alpha(n, k))^{2}\left|a_{k-1}\right|^{2} & \leq(A-B)^{2} p^{2}-\left(1-B^{2}\right) \sum_{j=1}^{k-1}(j-p)^{2}(\alpha(n, j))^{2}\left|a_{j-1}\right|^{2} \\
& \leq(A-B)^{2} p^{2} .
\end{aligned}
$$

Hence

$$
\left|a_{k-1}\right| \leq \frac{p(A-B)}{(k-p) \alpha(n, k)} .
$$

The estimate is sharp for the function $f(z)$ given by

$$
-z^{p+1}\left(D^{n+p-1} f(z)\right)^{\prime}=p \frac{1+A z^{k}}{1+B z^{k}}, \quad k=1,2 \ldots
$$


Further we obtain a sufficient coefficient condition, for a function to be in 1.1 ages $\bigcup_{n, p}(A, B)$ when $-1 \leq B<0$.

Theorem 5. Let the function $f(z)=\frac{1}{z^{p}}+\frac{a_{0}}{z^{p-1}}+\frac{a_{1}}{z^{p-2}}+\ldots$ be regular in $E$ and $-1 \leq B<0$. If

$$
\sum_{k=1}^{\infty}(k-p)(1-B) \alpha(n, k)\left|a_{k-1}\right| \leq p(A-B)
$$

where $\alpha(n, k)=\left(\begin{array}{c}n+p+k-1 \\ n+p-1\end{array}\right)$, then $f \varepsilon C_{n, p}(A, B)$. The result is sharp.

Proof. Suppose (19) holds. Then, for $z \varepsilon \Delta$, we have

$$
\begin{aligned}
& \left|z^{p+1}\left(D^{n+p-1} f(z)\right)^{\prime}+p\right|-\left|A p+B z^{p+1}\left(D^{n+p-1} f(z)\right)^{\prime}\right| \\
= & \left|\sum_{k=1}^{\infty}(k-p) \alpha(n, k) a_{k-1} z^{k}\right|-\left|(A-B) p+B \sum_{k=1}^{\infty}(k-p) \alpha(n, k) a_{k-1} z^{k}\right| \\
\leq & \sum_{k=1}^{\infty}(k-p) \alpha(n, k)\left|a_{k-1}\right| r^{k}-\left\{(A-B) p+B \sum_{k=1}^{\infty}(k-p) \alpha(n, k)\left|a_{k-1}\right| r^{k}\right\} \\
< & \sum_{-1}^{\infty}(k-p) \alpha(n, b)\left|a_{k-1}\right|-(A-B) p-B \sum_{k=1}^{\infty}(k-p) \alpha(n, k)\left|a_{k-1}\right| \\
= & \sum_{k=1}^{\infty}(k-p)(1-B) \alpha(n, k)\left|a_{k-1}\right|-(A-B) p \\
\leq & 0 .
\end{aligned}
$$

It follows that

$$
\left|\left\{z^{p+1}\left(D^{n+p-1} f(z)\right)^{\prime}+p\right\}\right| /\left|\left\{A p+B z^{p+1}\left(D^{n+p-1} f(z)\right)^{\prime}\right\}\right|<1
$$

It is easy to see that the inequality (20) is equivalent to (1). Hence $\int \varepsilon C_{n, p}(A, B)$.

The estimate is sharp for the function

$$
f(z)=\frac{1}{z^{p}}+\frac{P(A-B)}{(k-p)(1-B) \alpha(n, k)} z^{k-p}, \quad k=1,2, \ldots
$$

For this function

$$
\left|\left\{z^{p+1}\left(D^{n+p-1} f(z)\right)^{\prime}+p\right\}\right| /\left|\left\{A p+B z^{p+1}\left(D^{n+p-1} f(z)\right)^{\prime}\right\}\right|=1 \text {, for } z=1
$$


and the equality is attained in (19).

Remark The converse of the above theorem need not be true. Consider the function

$$
\begin{gathered}
f(z)=\frac{1}{z^{p}}+\frac{a_{0}}{z^{p-1}}+\frac{a_{1}}{z^{p-2}}+\ldots \text { where } \\
-z^{p+1}\left(D^{n+p-1} f(z)\right)^{\prime}=p \frac{1+A z}{1+B z}, \quad-1 \leq B<0, \quad z \varepsilon \Delta .
\end{gathered}
$$

Clearly $f \varepsilon C_{n, p}(A, B)$. Also it is easy to verify that

$$
a_{k-1}=-\frac{(A-B) p(-B)^{k-1}}{(k-p) \alpha(n, k)} .
$$

Hence

$$
\sum_{k=1}^{\infty}(k-p)(1-B) \alpha(n, k)\left|a_{k-1}\right|=(A-B) p \sum_{k=1}^{\infty}(1-B)(-B)^{k-1}>(A-B) p .
$$

The result follows.

Finally we state the following closure theorem for the class $C_{n, p}(A, B)$, the proof of which is obvious.

Theorem 6. If the functions $f$ and $g$ belong to the class $C_{n, p}(A, B)$ and $0 \leq s \leq 1$, then the function $F$ defind by $F(z)=s f(z)+(1-s) g(z)$ also belongs to $C_{n, p}(A, B)$.

\section{References}

[1] M. D. Ganigi and B. A. Uralegaddi, "Subclasses of meromorphic close-to-convex functions," Bull. Math. Soc. Sci. Math. R. S. Roumanie (N.S.) 33(81) (1989), No. 2 105-109.

[2] I. S. Jack, "Functions Starlike and convex of order $\alpha$," J. London Math. Soc. (2) 3 (1971), 469-474.

[3] Vinod Kumar and S. L. Shukla, "Multivalent functions defined by Ruscheweyh Derivatives-II," Indian J. Pure appl. Math. 15(11) (1984), 1228-1238.

Department of Mathematics, Karnatak University, Dharwad-580 003, India. 